\title{
Trascendencia, mundo y libertad en el entorno de Ser y Tiempo de Martín Heidegger
}

\author{
ENRIQUE MUÑOZ PÉREZ \\ Universidad Católica del Maule (Chile) \\ enmunoz@ucm.cl
}

\begin{abstract}
Resumen
El presente artículo busca mostrar algunos de los fundamentos de una de las tesis más conocidas del entorno de Ser y Tiempo: «el ser humano es formador de mundo» (Der Mensch ist weltbildend). Propongo que con la caracterización del Dasein como «trascendente» (Transzendenz), Heidegger quiere acentuar que el Dasein presenta una determinación fundamental que es formadora de mundo, fundada en la «libertad» (Freibeit).
\end{abstract}

Palabras claves: Ser humano, mundo, libertad, trascendencia.

\section{Transcendence, world and freedom in the environment of Being and Time of Martin Heidegger}

\begin{abstract}
This article examines some of the fundaments of one of the best known thesis in the environment of Being and Time: «the man is forming world» (Der Mensch ist weltbildend). I propose that the characterization of Dasein as «transcendence» (Transzendenz), Heidegger wants to emphasize that Dasein has a fundamental determination that is forming world, founded on «freedom» (Freiheit).
\end{abstract}

Key words: Human being, world, freedom, transcendence.

Doctor en Filosofía por la Universidad de Friburgo, Alemania. Profesor Adjunto del Departamento de Filosofía de la Facultad de Ciencias Religiosas y Filosóficas de la Universidad Católica del Maule. Es autor de los libros Der Mensch im Zentrum, aber nicht als Mensch. Zur Konzeption des Menschen in der ontologischen Perspektive Martin Heideggers (2008) y Heidegger y Scheler. Estudios sobre una relación olvidada (2013). Además, cabe mencionar los artículos recientes: "El rol del amor en la construcción de una ética fenomenológica" (2010), "Planteamientos ante la intersubjetividad: el Sympathiebuch de Max Scheler y su repercusión en Ser y Tiempo de Martin Heidegger" (2011), y "Fuentes fenomenológicas de la noción de persona: su discusión en Husserl, Scheler y Heidegger" (2012). Actualmente se desempeña como Director de Investigación de la Universidad Católica del Maule.

Este artículo fue presentado en la V. Messkircher Heidegger Konferenz, "Freiheit und Geschick", que se desarrolló entre el 22 y el 25 de mayo de 2014, en Messkirch, Alemania. 


\section{Introducción}

El presente artículo busca mostrar algunos de los fundamentos de una de las tesis más conocidas de la lección Los conceptos fundamentales de la metafisica. Mundo, finitud, soledad (1929/30) de Martin Heidegger. La tesis de Heidegger es: «el ser humano es formador de mundo» (Der Mensch ist weltbildend). Ella es tematizada por Heidegger para diferenciar la relación que tiene el ser humano con el mundo, en comparación con la piedra y el animal. El punto que me interesa abordar, en esta ocasión, es que Heidegger, en la lección del 29/30, no hace referencias explícitas a los supuestos que le permiten sostener la mencionada tesis sobre el ser humano, por lo que ellos tienen que ser rastreados en el entorno de Ser y Tiempo, esto es, en aquellas lecciones y conferencias que fueron desarrolladas por Heidegger entre 1927 y 1932, período al que pertenece también la lección del 29/30. Considero significativos para desentrañar dichos supuestos los siguientes textos de Heidegger: las lecciones magistrales Principios metafísicos de la lógica (1928) e Introducción a la filosofía $(1928 / 29)$. También es importante el escrito dedicado en homenaje a Husserl titulado De la esencia del fundamento (1929).

El contexto en el que Heidegger trata el tema de la libertad en el entorno de su obra fundamental, está marcado profundamente por el «fracaso» del proyecto de Sery Tiempo o, al menos, por su carácter inconcluso (Figal, 1999: 93). Heidegger intenta ahora una nueva aproximación al problema del sentido del ser y, precisamente por ello, reelabora algunos conceptos fundamentales de Ser y Tiempo. Las últimas lecciones de Marburgo y las primeras que Heidegger desarrolla en Friburgo como sucesor de Husserl, permiten constatar que son tres sus principales problemáticas en ese período: la pregunta por la esencia de la libertad, la pregunta por la esencia del fundamento y la pregunta por la esencia de la verdad ${ }^{1}$. En el presente artículo me enfocaré únicamente en la primera de estas preguntas, procurando responder la siguiente interrogante: ¿cuáles son las más importantes determinaciones del Dasein que son reformuladas o radicalizadas en el entorno de Ser y Tiempo? Mi hipótesis al respecto es la

1 La primera de estas preguntas Heidegger la aborda a través de una exhaustiva interpretación de la Crítica de la Razón Pura y de la Crítica de la razón práctica que lleva a cabo tanto en Interpretación fenomenológica de la Crítica de la razón pura de Kant (1927/28) e Introducción a la filosofía (GA 31); la segunda problemática Heidegger la aborda en Principios metafísicos de la lógica (1928); y la última, entre muchos textos, en diálogo con Platón, por ejemplo, en De la esencia de la verdad (1930). Al respecto, véase el excelente artículo de Alejandro G. Vigo: "Verdad, libertad y trascendencia en Heidegger. La radicalización de un motivo central de Sein und Zeit en los escritos de los años 1929-1930", citado al final en las Referencias. 
siguiente: En el entorno de Ser y Tiempo, Heidegger sigue orientando su trabajo por la problemática del estar-en-el-mundo como constitución fundamental del Dasein. Sin embargo, en este contexto, él intenta abordar, de otra manera, la estructura de la «trascendencia» (Transzenden $\approx$ ). Con la caracterización del Dasein como «trascendente» Heidegger quiere acentuar, entonces, que el Dasein presenta una determinación que es formadora de mundo. En esa línea de interpretación es posible constatar los fundamentos del tratamiento del tema de la «libertad» (Freibeit), que constituye el andamiaje de la afirmación de la lección del 29/30, según la cual el ser humano es formador de mundo.

En consecuencia, el presente artículo se divide en las siguientes partes: 1. La trascendencia en el entorno de Sery Tiempo, 2. Mundo y libertad en el entorno de Ser y Tiempo y 3. El ser humano como configurador de mundo en Los conceptos fundamentales de la metafísica. Mundo, finitud, soledad (1929/30).

\section{La trascendencia en el entorno de Ser y Tiempo}

Comenzaré a desarrollar la mencionada hipótesis a partir de la autointerpretación que desarrolla Heidegger de su obra principal, expuesta en Principios metafísicos de la lógica (1928). En esta lección, donde Heidegger tiene como interlocutor a Leibniz, aborda algunas de las críticas que se hicieron en el marco de la recepción de Ser y Tiempo. Especial importancia tiene, para el presente trabajo, el parágrafo $\$ 17$ donde él tematiza «El problema de la trascendencia y el problema de Ser y Tiempo». Dicho problema, a su juicio, encuentra su punto de partida en «la ontología fundamental como analítica de la existencia del Dasein. (...) A partir del modo de ser del Dasein, se trae a la luz la comprensión del ser» (Heidegger, 2007: 160). Heidegger desarrolla, en doce tesis, una respuesta a las primeras interpretaciones de Ser y Tiempo desde la perspectiva antropológica y ética, reafirmando sus principales planteamientos. No es este el lugar para profundizar en ellas, aunque constituyen el marco a partir del cual surge el tema de la trascendencia. Heidegger busca reiterar cuál es el objetivo de su proyecto filosófico, que no es otro que «mostrar la intrínseca posibilidad de la comprensión del ser y esto quiere decir, a la vez, la de la trascendencia» (Heidegger, 2007: 166).

$\mathrm{La}$ «trascendencia» (Transzenden $)$ es, sin duda alguna, una de las problemáticas centrales del entorno de Ser y Tiempo, constituyéndose en uno de los conceptos claves de ese período. Como es conocido, Heidegger desarrolló la problemática del «estar-en-el-mundo»(In-der-Welt-seins) en Ser y Tiempo, bajo la perspectiva del ente intramundano. En esta ocasión, Heidegger privilegia el abordaje del tema del mundo desde el prisma de 
la trascendencia, la que presenta una estrecha relación con el problema del ser. Dicho más precisamente: «Que 'el Dasein trasciende' quiere decir que configura un mundo en la esencia de su ser» (Heidegger, 2001: 136). Esta es, como espero mostrar, la característica decisiva de la noción de trascendencia en el pensamiento de Heidegger de este período. Antes de seguir profundizando en esta idea planteada en De la esencia del fundamento (1929), quisiera presentar el contexto en el que emerge dicha afirmación.

En Principios metafísicos de la lógica (1928), Heidegger analiza, en primer lugar, la tradicional relación sujeto-objeto, donde se enraíza el problema de la trascendencia. En otras palabras, Heidegger busca mostrar que la trascendencia tiene una estructura ontológica. Así lo explica Heidegger: «Toda la problemática hasta ahora vigente de la 'relación' del 'sujeto' y el 'objeto' se encuentra no discutida en la base del problema de la trascendencia, entiendo esta expresión primariamente de un modo totalmente corriente: un ente (el Dasein) asciende hasta otro ente (Dasein o subsistente) de un modo tal que, en este trascender, aquello hacia lo que el Dasein trasciende es para él develado en un sentido muy amplio» (Heidegger, 2007: 158) Es decir, el análisis del problema de la trascendencia se ha reducido, en el entendimiento vulgar, a cómo se relaciona el «sujeto» y el «objeto». Según ello, no es posible entender un «sujeto» desligado de un «objeto» y viceversa. Lo que Heidegger quiere puntualizar, entonces, es que el problema de la trascendencia no puede ser comprendido bajo la lógica «sujeto-objeto», porque éste no es un problema de la teoría del conocimiento, sino que de la ontología. «Este problema de la trascendencia no se identifica con la relación de sujeto-objeto, sino que depende, de un modo más originario cuantitativa y cualitativamente, de manera inmediata del problema del ser en general» (Heidegger, 2007: 159). Para Heidegger, en consecuencia, el problema del ser y el problema de la trascendencia tienen una estrecha relación.

Aclarado este punto, Heidegger aborda el concepto «trascendencia» desde su perspectiva terminológica: «El significado de la palabra proviene de trascendere: pasar al otro lado, superar, ir más allá de hacia. Por consiguiente, trascendencia significa: paso al otro lado, pasar al otro lado y lo trascendente designa aquello hacia donde se produce el paso, aquello que, para ser accesible y comprensible, requiere un paso al otro lado, lo de más allá, lo contrapuesto: por último, lo que trasciende: aquello que realiza el paso más allá» (Heidegger, 2007: 188). No obstante lo anterior, Heidegger no persigue quedarse en el significado etimológico de la palabra «trascendencia», sino que a partir de ello, quiere alcanzar el nivel filosófico. En ese marco, distingue dos sentidos de la noción de «trascendencia»: «Este término significa: primero, lo trascendente a diferencia de lo inmanente; segundo, lo trascendente a diferencia de lo contingentes) (Heidegger, 
2007: 189). A su juicio, estos sentidos del término «trascendencia» representan dos líneas fundamentales de su interpretación: la epistemológica y la teológica, respectivamente. En el primer caso, la «trascendencia» es comprendida como lo que no permanece al interior del sujeto, sino que está fuera suyo; en el segundo caso, ella es entendida como «lo incondicionado, pero, a la vez, lo propiamente inalcanzable: lo que nos excede» (Heidegger, 2007: 190). No obstante lo anterior, Heidegger considera que ambas posiciones están orientadas equivocadamente. Antes que una aproximación epistemológica o teológica, la "trascendencia" es primero, «la constitución originaria de la subjetividad de un sujeto. El sujeto trasciende qua sujeto, no sería sujeto si no transcendiese. Ser sujeto quiere decir transcender» (Heidegger, 2007: 195). De esta manera, Heidegger salda una deuda pendiente que tenía relación con el fundamento ontológico de la subjetividad ${ }^{2}$. Dicho en dos palabras, «ser sujeto quiere decir transcenden» (Heidegger, 2007: 195). En otras palabras, el Dasein no existe y luego, en algunas ocasiones, efectúa una superación, sino que existir significa para Heidegger, originariamente, «superar» (übersteigen). En resumen, ni la perspectiva epistemológica ni la perspectiva teológica apuntan en la dirección correcta, puesto que el problema de la trascendencia tiene que ver con la constitución esencial del Dasein, pertenece primariamente a su ser y no sólo es un comportamiento añadido. Heidegger designa, por consiguiente, el fenómeno fundamental de la trascendencia con una expresión conocida: «ser-en-el-mundo» (Heidegger, 2007: 196). En lo que sigue, desarrollaremos brevemente el tema del mundo para vincularlo con el tópico de la libertad.

\section{Mundo y libertad en el entorno de Ser y Tiempo}

En este apartado, quisiera mostrar el vínculo que existe para Heidegger entre las nociones de «Dasein», «mundo» $\mathrm{y}$ «trascendencia» como antecedente del tema de la libertad. Ello me obliga, en primer lugar, a esbozar cómo entiende ahora Heidegger la noción de mundo y cuáles son las novedades en comparación con Sery Tiempo. Como es sabido, el tema del «mundo» en Sery Tiempo es abordado desde la perspectiva de la estructura fundamental del existir humano que es el «estar-en-el-mundo». Hei-

2 En De la esencia del fundamento (1929) Heidegger refuerza esta idea: «Si elegimos para este ente que nosotros mismos somos y que entendemos como 'Dasein' el título de 'sujeto', entonces la trascendencia designa la esencia del sujeto y es la estructura fundamental de la subjetividad. Pero no es que el sujeto exista previamente como 'sujeto' y después, si se da el caso, también se presenten objetos que tienen que ser trascendidos, sino que ser sujeto significa: ser ente en y como trascendencia» (Heidegger, 2001: 120). 
degger sostiene que el «mundo» es una constitución fundamental de la existencia humana y, por consiguiente, no de las cosas o seres intramundanos. Él afirma, por ejemplo, que «no hay algo así como un 'estarjuntos' del ente llamado 'Dasein' con otro ente llamado 'mundo'» (Heidegger, 2012: 76). No hay dos entes porque el mundo no es un ente, sino la constitución de ser de ese ente que es el Dasein (Lambert, 2006: 45-54).

En el entorno de Ser y Tiempo, el problema del mundo es tematizado desde otra perspectiva: la trascendencia. No existe una contradicción entre la obra principal de Heidegger y los textos que componen el entorno de Ser y Tiempo, sino que un enfoque distinto: «Si la trascendencia en el sentido del ser-en-el-mundo es la constitución metafísica fundamental del Dasein, entonces una metafísica del Dasein, con suficiente intención ontológica fundamental, debe señalar esta constitución fundamental desde el principio» (Heidegger, 2007: 196) En otras palabras, el giro «ser-en-el-mundo», esto es, en último término el «mundo», tiene que ser considerado en relación al problema del horizonte de la trascendencia, pero en el marco de un lugar filosófico novedoso: la metafísica del Dasein.

Abordemos brevemente la noción de «metafísica del Dasein» (Metaphysik des Daseins) para después proseguir con el tema de la «trascendencia». Su tratamiento nos exige volver al texto Kant y el problema de la metafísica que Heidegger publicara en el año 1929 y que corresponde al entorno de Ser y Tiempo. En ese texto, Heidegger modifica la noción de «ontología». Ella ya no es la disciplina que se dedica a desentrañar las estructuras fundamentales del Dasein, como es descrita en Ser y Tiempo, sino que tiene un carácter propedéutico en relación a la metafísica. Más precisamente, la ontología fundamental es entendida ahora como aquella «analítica ontológica de la esencia finita del hombre que debe preparar el fundamento de una metafísica "conforme a la naturaleza del hombre"”. (Heidegger, 1996: 11). Este ajuste en el pensamiento heideggeriano tiene que ver, como ya he dicho, con su desencanto ante la recepción de Sery Tiempo, lo que lo impulsa a reorientar su investigación a través del análisis de una de las obras fundamentales de Kant: la Crítica de la razón pura. En ese contexto, la tarea de la "metafísica del Dasein", según Heidegger, es la compresión del fundamento propio del hombre, que es esencialmente finito ${ }^{3}$.

3 «Si el hombre sólo es hombre a raíz del ser-abí/Dasein en él, la pregunta por lo que es más primordial que el hombre no puede ser, en principio, una pregunta antropológica. Toda antropología, aun la filosófica, supone ya al hombre como hombre. El problema de la fundamentación de la metafísica se enraíza en la pregunta por el ser-ahí/Dasein en el hombre, es decir, en la pregunta por su fundamento íntimo, por la comprensión del ser como finitud esencialmente existente» (Heidegger, 1996: 193). 
Una vez hecha esta aproximación a la «metafísica del Dasein», corresponde seguir profundizando el análisis del mundo bajo la perspectiva de la «trascendencia». Una de las constataciones que realiza Heidegger en Introducción a la filosofía (1928/29) y que también se encuentra en el texto paralelo De la esencia del fundamento (1929), es que el término «mundo» es ambiguo. De este modo, en el \$33 de la mencionada lección magistral, Heidegger se pregunta por el significado de «mundo»: «En las consideraciones históricas que he hecho en De la esencia del fundamento (Heidegger,

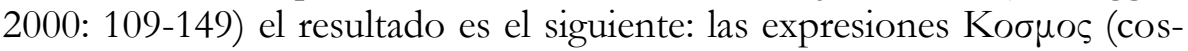
mos), mundus, mundo, tienen un doble significado: un significado vulgar, el ente en tanto que naturaleza, y un significado filosófico, que, sin embargo, conceptualmente no está articulado de forma expresa» (Heidegger, 2001: 253). De ahí puede deducirse que Heidegger se distancia de una comprensión superficial del concepto de «mundo», por ejemplo, en la línea de «mundo» como lo «óntico-natural», como una «región naturaleza» o como término del lenguaje común, por ejemplo, cuando es usado en giros como «dama de mundo» $\mathrm{u}$ «hombre de mundo» (Heidegger, 2007: 211).

Por el contrario, Heidegger busca enfatizar que «mundo» «caracteriza la suma total de los entes en la totalidad de sus posibilidades» (Heidegger, 2007: 211), la que está esencialmente referida a la existencia humana y a su carácter finito. Esta idea del «mundo como totalidad» se encuentra también en otros textos del entorno de Ser y Tiempo donde Heidegger habla de «mundo» como «el cómo del ente en su conjunto» (Heidegger, 2001:253) de igual forma referido al Dasein o de «mundo» como la relación que establece la existencia humana «con lo ente en su totalidad. (...) El mundo pertenece a una estructura de referencia que caracteriza al Dasein como tal, y a la que llamamos ser-en-el-mundo» (Heidegger, 2000: 134). Resumiendo, es posible decir que, para Heidegger, el mundo determina el horizonte de la trascendencia, esto significa que el «mundo» no es la agrupación de un conjunto de entes, sino que aquel horizonte donde se presenta el ente a la existencia humana. En otras palabras, el concepto ontológico de «mundo» caracteriza la esencia metafísica del Dasein, cuya constitución fundamental es la «trascendencia». La «trascendencia», en consecuencia, está formada por tres aspectos fundamentales: a) el «sobrepasar» (das Übersteigen) de «algo hacia algo» que lleva a cabo el Dasein; b) el «para-qué» (woraufzu), es decir, el mundo y c) en la trascendencia «algo» (etwas) es superado, esto es, el ente. En otras palabras, para Heidegger, «Dasein», «mundo» y «trascendencia» se presentan en una estrecha relación, teniendo como núcleo de ella a la «libertad» (Freibeit). De este modo, sostiene Heidegger, «en la medida en que la trascendencia, el seren-el-mundo, constituye la estructura fundamental del Dasein, el ser-en- 
el-mundo debe estar también originariamente unido a la determinación fundamental de la existencia del Dasein, es decir, a la libertad o surgir de ella» (Heidegger, 2007: 217). Dicho brevemente: para Heidegger la trascendencia y la libertad son idénticas. Sólo donde hay libertad, hay un «por mor de» (Umwillen), hay mundo. O dicho de otra manera, un ente en tanto que es libre es, «en sí mismo, necesariamente un ente que trasciende» (Heidegger, 2007: 217).

¿Qué es, entonces, libertad para Heidegger? La libertad no es entendida en este período como «espontaneidad» o como algún modo de «causalidad» o «empezar-por-sí-mismo» (Heidegger, 2000: 140), sino que tiene que ser interpretada en un sentido metafísico (Heidegger, 2007: 224). Heidegger acentúa, en primer lugar, que pertenece a la esencia ontológica del Dasein el «por mor de» (Umwillen). Dicho «por mor de» es «aquello bacia lo que el Dasein, en tanto que trasciende, trasciende» (Heidegger, 2007: 223) o con otros términos, el «por mor de» es «la intrínseca posibilidad de la voluntad» (Heidegger, 2007: 223). Voluntad no tiene que ser entendida aquí como un mero acto del sujeto a diferencia de otros actos como el imaginar o el enjuiciar, sino que en una dimensión ontológica: la intrínseca posibilidad de la voluntad, de la que habla Heidegger, es la libertad. «En la esencia de la libertad se encuentra este darse el por mor de» (Heidegger, 2007: 223).

En otras palabras, la libertad es la condición estructural de la existencia humana que le permite llevar a cabo o no llevar a cabo sus posibilidades, de efectuar o no efectuar sus proyectos, en último término, de formar no o no formar mundo. El mundo, sostiene Heidegger, «nunca es, sino que se hace mundo» (Heidegger, 2000: 140) y allí la libertad muestra su carácter vinculante: la libertad es la única que puede lograr que el mundo se haga mundo para el Dasein. O con palabras de Heidegger:

La libertad hace al Dasein, en el fundamento de su esencia, vinculable consigo mismo; con mayor precisión: la da a sí mismo la posibilidad del vínculo. El todo del vínculo que se encuentra en el por mor de es el mundo (Heidegger, 2007: 224).

A partir de este fundamento es posible entender mejor, por un lado, el sentido que tiene la «trascendencia» y el «mundo» en este período del pensamiento de Heidegger posterior a Ser y Tiempo. El «mundo» es la realización de las posibilidades del Dasein, las que él mismo sobrepasa. El Dasein tiene la capacidad de formar o configurar el mundo, porque éste no está dado o, como afirma Heidegger, «el Dasein, en cuanto libre, es proyecto del mundo» (Heidegger, 2007: 224). Se puede apreciar, entonces, el despliegue del fundamento ontológico de la expresión que dio 
origen a este trabajo: «el ser humano es formador de mundo» (Der Mensch als weltbildend) y agregar que el ser humano es configurador de mundo porque es ontológicamente libre. Me resta, por último, vincular estos supuestos con la lección del 29/30.

\section{El ser humano como configurador de mundo en Los conceptos fundamentales de la metafísica. Mundo, finitud, soledad $(1929 / 30)^{4}$}

Un par de años después de la publicación de Ser y Tiempo y en medio de su polémica recepción, Heidegger dicta la lección Los conceptos fundamentales de la metafísica. Mundo, finitud, soledad (1929/30). En este texto Heidegger se aboca a describir, en su primera parte, un estado de ánimo fundamental: «el aburrimiento» (die Langeweile). El mencionado temple de ánimo debiera inspirar el quehacer filosófico en medio de la compleja situación que vive la humanidad a fines de los años veinte del siglo pasado: las consecuencias de la Primera Guerra Mundial, la Gran Depresión, la falta de sentido de la vida, etc. Heidegger distingue, por consiguiente, tres estadios del aburrimiento: «el ser aburrido por algo» (das Gelangweiltwerden von etwas) (Heidegger, 2007:111), «el aburrirse con algo» (das Sichlangweilen bei etwas) (Heidegger, 2007:143) y «el aburrimiento profundo como el 'uno que se aburre'» (die tiefe Langeweile als das «es ist langweilig) (Heidegger, 2007:173). En otras palabras, Heidegger busca subrayar con la descripción fenomenológica de este estado de ánimo fundamental, en especial con el tercer estadio, que la filosofía debe acometer una tarea urgente e ineludible: la «liberación del Dasein en el ser humano» (die Befreiung des Daseins im Menschen) ${ }^{5}$.

Por otra lado, en la segunda parte de la lección, Heidegger une la descripción del temple de ánimo fundamental, el aburrimiento, con las preguntas principales de su concepción metafísica; esto es, la pregunta por la soledad, por la finitud y por el mundo. Heidegger focaliza su atención de modo primordial en esta última pregunta.

Este apartado es un versión mejorada de la segunda parte de mi artículo "Ser humano, animal y animalidad: Novedad y alcance de Los Conceptos fundamentales de la metafísica. Mundo, Finitud, soledad 1929/30 de Martin Heidegger" publicado en esta misma revista (Muñoz, 2013).

5 Heidegger, 2007:220. El traductor de Los conceptos fundamentales de la metafísica, Alberto Ciria, traduce el giro «die Befreiung des Daseins im Menschen» como «la liberación de la existencia en el hombre». Si bien es una traducción literalmente correcta, no hace juicio filosófico a la aparición de la mencionada expresión una vez sostenidas todas las críticas de Heidegger tanto a la antropología filosófica como al concepto de ser humano que acaece en Sery Tiempo y en Kant y el problema de la metafísica. 
Con todo, antes de responderla, él hace la siguiente consideración metodológica. Para responder la pregunta por el mundo, no hay que recurrir a una interpretación fundada en los entes intramundanos, al modo de Ser y Tiempo; tampoco se debe desarrollar una aproximación histórica al concepto de mundo, tal como lo hizo en De la esencia del fundamento (1929), sino que llevar a cabo una «consideración comparativa» (Heidegger, 2007: 227). Heidegger sostiene que «el hombre tiene mundo» (Heidegger, 2007: 227) Empero, ¿qué sucede con los otros entes, por ejemplo los animales, las plantas, las cosas materiales, las piedras? Él responde a esta interrogante formulando las tres conocidas tesis: «1) la piedra (lo material) es sin mundo, 2) el animal es pobre de mundo; 3) el ser humano configura mundo» (Heidegger, 2007: 227) ${ }^{6}$.

Dicho de otra manera, Heidegger persigue con la discusión comparativa de las tres tesis «delimitar provisionalmente lo que entendemos en general por el título mundo» (Heidegger, 2007: 235). Si esto es así, ¿qué es, entonces, el mundo? Al inicio de la segunda parte de la lección, Heidegger nos entrega la siguiente pista. Él sostiene que llamamos «mundo» a «esta amplitud de este 'en conjunto' que se manifiesta en el aburrimiento profundo» (Heidegger, 2007: 217) En la disposición afectiva que llamamos «aburrimiento profundo» se nos manifiesta como seres humanos una totalidad, un conjunto o la suma de lo ente accesible al que denominamos «mundo». Sin embargo, esta manifestabilidad de lo ente no es una exclusividad del ser humano, sino que también está al alcance del animal. El punto para Heidegger estriba en que la accesibilidad de lo ente no es la misma en el caso del ser humano que en el animal; ella es «modificable según el alcance y la profundidad del penetramiento» (Heidegger, 2007: 244) A propósito de este último punto, Heidegger es claro al sostener que él no busca explicar cómo animales y seres humanos se diferencian en algún aspecto específico, al modo de la antropología física, sino que su finalidad es esclarecer «qué constituye la esencia la animalidad del animal y la esencia de la humanidad del hombre» (Heidegger, 2007: 228).

De este modo, vuelvo sobre el análisis heideggeriano de las tres tesis antes mencionadas. Dicho análisis se concentra principalmente en la delimitación de las diferencias esenciales entre el animal y el ser humano, en el marco de una nueva aproximación al fenómeno del mundo. Dichas diferencias son tres: el «ponerse en el lugar del otro», el «comportamien-

\footnotetext{
6 Es interesante hacer notar cómo esta «consideración comparativa» heideggeriana entre el hombre, el animal y la piedra, es en gran medida una respuesta ontológica a los planteamientos antropológicos de Max Scheler (El puesto del hombre en el cosmos, 1928) y de Helmuth Plessner (Die Stufen des Organischen und der Mensch, 1928).
} 
to» y la estructura del «en tanto que». A propósito de esta última aparece el tema de la libertad en la lección del 29/30.

De este modo, Heidegger sostiene, en primer lugar, que la diferencia entre el ser humano y el animal se encuentra en la capacidad que tiene el hombre de «ponerse en el lugar del otro» o «poder transponerse en otros» o el «transponerse» (das Sichversetzenkönnen), cuestión que al animal le está vedada. Así lo sostiene Heidegger: «En la medida en que un ser humano existe, en tanto que existente está ya transpuesto en otros seres humanos, aun cuando de hecho no haya ningún otro ser humano cerca. Ser-ahí el ser humano (Da-sein des Menschen), ser-ahí en el ser humano (Da-sein im Menschen), significa por tanto -no exclusivamente, pero sí entre otras cosas- estar transpuesto en otros seres humanos (das Versetztsein in andere Menschen). El poder transponerse en otros seres humanos como acompañarlos a ellos (Das Sichversetzenkönnen in anderen Menschen als Mitgehen mit ihnen), a la existencia en ellos, sucede ya siempre en función de la existencia del ser humano... en cuanto existencia» (Heidegger, 2007: 256).

$\mathrm{El}$ «ser humano» puede ponerse en lugar de otro «ser humano», puede empatizar y hacer amistad con otro, puede sufrir las penas del otro o alegrarse con sus éxitos; cuestión que al animal no le es posible. Un ejemplo grafica, según Heidegger, las limitaciones del animal: los animales domésticos. El animal doméstico -un perro, un gato o un pequeño conejo- puede comer en un plato, tener una casa o trepar la escalera. «Los animales domésticos los tenemos en casa, 'viven' con nosotros. Pero nosotros no vivimos con ellos, si es que vivir significa ser al modo del animal. No obstante, estamos con ellos. Pero este ser-con tampoco es un coexistir, en la medida en que un perro no existe sino que simplemente vive» (Heidegger, 2007: 261) Heidegger hace presente de esta manera que en el animal no sólo no hay transponibilidad, al modo de los seres humanos, sino que el animal tiene una relación con el mundo que es pobre. Es cierto que el perro trepa las escaleras, el gato come en un plato o que el conejo vive en una pequeña casa, pero ni el perro ni el gato ni el conejo se comportan respecto de la escalera, del plato o de la pequeña casa «en tanto que» (als) tal. En el animal hay, por consiguiente, un tener y no tener mundo.

El animal muestra en él mismo una esfera de la transponibilidad (Versetzbarkeit) a él o, dicho más exactamente: él mismo es esta esfera, que sin embargo deniega un acompañar. El animal tiene una esfera de la transponibilidad (Versetzbarkeit) posible en él, y no obstante no necesita tener eso que nosotros llamamos mundo. (...) El animal tiene algo y no tiene algo. Lo expresamos así: el animal es pobre de mundo, carece fundamentalmente de mundo (Heidegger, 2007: 262). 
¿Cómo se expresa la diferencia entre el modo de ser del animal y modo de ser del ser humano? Heidegger considera que el conducirse del animal es un «hacer/empujar» (treiben $)^{7}$ y que el comportarse del ser humano es un «actuar» (bandeln). Él nos presenta, en primer lugar, el ejemplo del topo y la lombriz: «La lombriz que huye no aparece simplemente en el contexto de una sucesión de movimientos que parten del topo, sino que buye de éste. Allí no se está desarrollando simplemente algo, sino que la lombriz que huye se conduce como huyente de una manera determinada frente a éste; y éste, el topo, por el contrario, se conduce frente a la lombriz en tanto que la persigue» (Heidegger, 2007: 289). En el fondo, el conducirse del animal es un «ser capaz de» (das Fäbigsein zu) -huir del topo o atrapar a la lombriz - «impulsivo» (triebhaft). Eso significa que el animal, conforme a su esencia, se conduce «en un medio circundante (Umgebung), pero jamás en un mundo (Welt)» (Heidegger, 2007:291) En otras palabras, el animal se encuentra encerrado en un anillo, que es su medio circundante, en el que se haya «cautivado en sí» (Heidegger, 2007: 291).

Por el contrario, el modo de ser del hombre es totalmente distinto, es «el comportarse con respecto a» (das Sichverbalten ₹u), el «hacer» (Tun) o el «actuar» (bandeln) en un mundo configurándolo, transformándolo o determinándolo. De este modo, en el marco del esclarecimiento de la tercera tesis sobre la consideración comparativa sobre el mundo, expondré la última diferencia fundamental entre el ser humano y el animal: el «en tanto que» (als).

Heidegger ya ha sostenido que el ser humano forma o configura mundo. El animal, en cambio, sólo tiene un acceso limitado al mundo o a lo ente. Heidegger lo ejemplifica de la siguiente manera: «El nido que se busca, la presa que se caza, después de todo no son una nada, sino que un ente, de otro modo el pájaro no se podría posar en el nido ni el gato cazar un ratón, si no fueran ente» (Heidegger, 2007: 324).

Es claro que el animal tiene un acceso concreto a algo que realmente es, «sin embargo, eso es algo que sólo nosotros somos capaces de experimentar y, ostensiblemente, de tener, en tanto que ente» (Heidegger, 2007: 324). Por ello es que en el animal hay un tener y un no tener mundo.

¿Qué quiere decir, entonces, «configuración de mundo»? Antes de clarificar esta expresión, Heidegger deja en claro que esta pregunta no corresponde al ámbito de la antropología filosófica, sino que una cues-

\footnotetext{
Me parece, en esta ocasión, no del todo afortunada la traducción del verbo treiben por «hacer». Como el mismo traductor sostiene treiben significa «impulsar», «mover»o, como me parece mejor, «empujar». El sustantivo Treiben se puede traducir como «movimiento», «animación»y «actividad». En último término, considero que Heidegger quiere mostrar que tras la conducta animal hay un impulso.
} 
tión de carácter ontológico-existencial: «(...) todo preguntar del hombre por el hombre es en primer término y en última instancia un asunto de la existencia respectiva del hombre. (...) La pregunta de qué sea el hombre, si se plantea realmente, entrega al hombre expresamente a $s u$ existencia» (Heidegger, 2007: 339) No obstante lo anterior, Heidegger reconoce en el parágrafo $\$ 67$ de Los conceptos fundamentales de la metafísica. Mundo, finitud, soledad (1929/30) que no ha dicho nada sustantivo respecto de la significación del giro "configuración de mundo» ${ }^{8}$. Hay que esperar para ello al parágrafo siguiente.

En el parágrafo $\int 68$ de la lección del 29/30 encontramos la primera clarificación explícita del giro «configuración de mundo», de hecho, el título del parágrafo da cuenta, por un lado, del concepto de mundo que utilizará Heidegger: «mundo es la manifestabilidad de lo ente en cuanto tal en su conjuntor (Heidegger, 2007: 343) y, por otro lado, de la finalidad del parágrafo mismo: aclarar de modo general la noción de configuración de mundo.

Heidegger comienza su análisis explicando el significado de la expresión «configuración de mundo». Según él, el mundo pertenece intrínsecamente a la configuración de mundo. «La manifestabilidad de lo ente en cuanto tal en su conjunto, el mundo, se configura, y mundo sólo es lo que es en tal configuración» (Heidegger, 2007: 344). Quien configura el mundo es el ser humano; pero, se pregunta Heidegger, de quién estamos hablando. No es ciertamente este ente el hombre corriente o de la calle, sino que el ser humano qua ser humano, o más precisamente, el «Dasein en el ser humano» (Dasein im Menschen) (Muñoz, 2007). Es en este contexto donde Heidegger define la expresión «configuración de mundo», advirtiendo su carácter polisémico:

‘Configuración de mundo’ lo empleamos intencionadamente en una multiplicidad de significados. La existencia en el hombre configura el mundo [ $\mathrm{Da}$ sein im Menschen ist weltbildend]: 1) lo produce; 2) da una imagen, una visión de él, lo representa; 3) lo destaca, es lo que lo abarca y lo rodea (Heidegger, 2007: 344).

En otras palabras, el ser humano no sólo produce artefactos o construye puentes, sino que forma con otros seres humanos su historia, su tiempo y su época o, si se prefiere, lleva a cabo sus proyectos. Con todo, este triple significado del configurar exige una interpretación más precisa

\footnotetext{
8 «Pero, después de todo, en la primera parte de la asignatura [de la lección] no hemos dicho nada de la esencia del hombre, ni menos aún de aquello que llamamos configuración de mundo y mundo» (Heidegger, 2007: 340).
} 
del fenómeno del mundo, esto es, una interpretación respecto de formas más básicas o prelógicas de acceso al ente (Vigo: 2003).

Dicho esto, Heidegger desarrolla inmediatamente la primera interpretación formal del «en tanto que» (als) como un momento estructural de la manifestabilidad del mundo?. Heidegger aborda, de este modo, la conexión del «en tanto que» (als) como la estructura de la relación y de los miembros de la relación con la proposición enunciativa. Más adelante, en este contexto, se referirá a la libertad y, nuevamente, al «concepto de configuración de mundo».

Heidegger comienza, en primer lugar, recapitulando lo expuesto. Se ha dicho que mundo es la manifestabilidad de lo ente en cuanto tal en su conjunto. Con esta caracterización emerge algo enigmático: este «en cuanto tal», lo ente en cuanto tal, algo en tanto que algo. $\mathrm{Y}$ he aquí lo relevante: «este 'en tanto que', que es totalmente elemental, es -así podemos decirlo simplemente- lo que le es negado al animal» (Heidegger, 2007: 346).

En otras palabras, el «en tanto que» (als) marca la diferencia entre lo animal y lo humano. El animal no puede representarse la «piedra como martillo» o tomar su vida "como un todo», al modo como lo hace el ser humano. He aquí la restricción en la penetrabilidad en el mundo del animal.

$\mathrm{El}$ «en tanto que» (als) está conectado, agrega Heidegger, en segundo lugar, con el problema conductor del mundo que está formulado en la tesis: el ser humano es configurador de mundo. Heidegger hace presente que no le interesa partir de una definición arbitraria y dada del ser humano, sino que busca, al menos, cuestionarla. Enfatiza, además, que desarrolla el problema del mundo desde un temple de ánimo fundamental: el aburrimiento. Con todo, cuando se aboca a aclarar el momento estructural del «en tanto que algo», Heidegger se percata que esta tarea no es tan evidente. Normalmente creemos que el «en tanto que» (als) es una relación. Sin embargo, esto que parece obvio, esconde un problema: «La base de la metafísica que hemos caracterizado, y su orientación con arreglo a la verdad de la proposición, aunque en cierto sentido es necesaria, sin embargo, no es original》 (Heidegger, 2007: 349).

Esta no-originalidad ha impedido, sostiene Heidegger, el correcto desarrollo del problema del mundo. En otras palabras, se hace necesario esclarecer la relación entre metafísica y lógica, cuestión que sobrepasa el

$9 \quad$ Hay que tener presente que el tratamiento de Heidegger sobre el tema del «en tanto que» (als) en la lección Los conceptos fundamentales de la metafísica. Mundo, soledad, finitud 1929/30 se enlaza con la comprensión de ser y con el horizonte de aperturidad del mundo en Sery Tiempo, particularmente en el \$32. 
objetivo del presente artículo. Baste con decir que el «en tanto que» (als), dado como un punto de partida del problema del mundo, es reconducido formalmente por Heidegger a la proposición enunciativa. $\mathrm{O}$, con sus palabras, «el 'en tanto que' guarda relación con el enunciado» (Heidegger, 2007: 396).

Si lo anterior es así, no es de extrañar que Heidegger relacione el giro «configuración de mundo» con el lógos y la libertad. Ello es desarrollado por Heidegger en el $\$ 73 \mathrm{c}$ de Los conceptos fundamentales de la metafísica. Mundo, finitud, soledad (1929/30). En ese lugar, él afirma que el lógos es «la potencia de un comportamiento que muestra lo ente, ya sea descubriendo (verdadero) u ocultando (falso). Esta potencia es como esta potencia sólo posible, si ella se fundamenta en un ser libre para lo ente en cuanto tali) (Heidegger, 2007: 401) ${ }^{10}$. O sea, el desocultamiento y el ocultamiento a través del enunciado, se abre recién allí como posibilidad donde impera la libertad (Vigo: 2003). De esta manera surge de nuevo, aunque brevemente el tema de la libertad, que se había presentado en otros textos del entorno de Ser y Tiempo. Dicho en dos palabras el lógos, como enunciado, sólo es posible allí donde hay libertad. «Descubrimiento y ocultamiento del lógos, verdad y ser falso, verdad $o$ falsedad, la posibilidad de ambas, sólo se dan donde hay libertad, y sólo donde hay libertad se da la posibilidad de la vinculatoriedad» (Heidegger, 2007: 403).

¿Y qué tiene que ver esto con la «configuración de mundo»? Más de lo que se creería a primera vista: el fundamento del lógos, la libertad, apunta a una manifestabilidad de lo ente que es previa a todo enunciar, que es prelógica y antipredicativa. Y este ámbito de manifestabilidad no es otro que el mundo y su configuración. De este modo, si el lógos «en cuanto a su posibilidad interna se remonta a algo más original, y si esto más original guarda alguna relación con lo que llamamos mundo y configuración de mundo, entonces los juicios y las proposiciones no son en sí mismos primariamente configuradores de mundo, aunque pertenecen a la configuración de mundo» (Heidegger, 2007: 403).

De este modo, que el ser humano configure mundo supone una cierta potencialidad, un estar abierto al ente mismo, un estar ofrecido al mundo, un cierto comportamiento original, etc.; en último término, que el ser humano configure mundo supone que es libre.

\footnotetext{
10 Me permito modificar levemente la traducción de Antonio Ciria, porque el texto en alemán es el siguiente: «Dieses Vermögen ist als dieses Vermögen nur möglich, wenn es gründet in einem Freisein für das Seiende als solches》 (Heidegger, 1992: 492).
} 


\section{REFERENCIAS}

-Figal, G. (1999). Martin Heidegger zur Einführung. Hamburgo: Junius.

-Heidegger, M. (1990). Metaphysische Anfangsgründe der Logik. Frankfurt a.M.: Vittorio Klostermann.

-Heidegger, M. (1992). Die Grundbegriffe der Metaphysik. Welt - Endlichkeit - Einsamkeit. Frankfurt a.M.: Vittorio Klostermann.

-Heidegger, M. (1996). Kant y el problema de la metafísica. México: Fondo de Cultura Económica.

-Heidegger; M. (2000). Hitos. Madrid: Alianza Editorial.

-Heidegger, M. (2001). Introducción a la filosofía. Valencia: Frónesis.

-Heidegger, M. (2007). Principios metafísicos de la lógica. Madrid: Síntesis.

-Heidegger, M. (2012). Ser y Tiempo. Madrid: Trota.

-Lambert, C. (2006). Mundo y existencia. Consideraciones fenomenológicas desde la perspectiva de Heidegger y $W$ elte. Santiago: Bricklediciones.

-Muñoz, E. (2007). Heidegger y la pregunta por el hombre. Veritas (16), 91-105.

-Muñoz, E. (2013). Ser humano, animal y animalidad: Novedad y alcance de Los Conceptos fundamentales de la metafísica. Mundo, finitud, soledad 1929/30 de Martin Heidegger. Veritas (29), 77-96.

-Vigo, A. (2003). Verdad, libertad y trascendencia en Heidegger. La radicalización de un motivo central de Sein und Zeit en los escritos de los años 1929-30. En P. Brickle (Ed.), La filosofía como pasión. Homenaje a Jorge Eduardo Rivera Cruchaga en su 75 cumpleaños. Madrid: Trotta.

Sumario: 1. La trascendencia en el entorno de Ser y Tiempo; 2. Mundo y libertad en el entorno de Ser y Tiempo; 3. El ser humano como configurador de mundo en Los conceptos fundamentales de la metafísica. Mundo, finitud, soledad 1929/30; Referencias. 